\section{Undergraduate Research and Climate Change}

\section{doi: $10.18833 /$ spur/5/1/3}

ᄂ

he signs of active climate change are everywhere. On June 29, 2021, the highest-ever temperature was recorded in Lytton, Canada: $121.4^{\circ} \mathrm{F}$; fire later decimated the village. The Bootleg fire in Oregon has been eclipsed by the Dixie fire in California as the largest in the United States; each has burned more than 400,000 acres. The death toll from July's catastrophic floods in Germany and Belgium hovers around 200. A flash flood in Zhengzhou, China, a city located on the Yellow River, trapped more than 500 subway riders for hours. Climate change is an exigent problem, presenting challenges in transforming energy systems and agricultural practices, rethinking political structures and international cooperation, and addressing climate-induced migration. Such a difficult and daunting reality requires educators to engage students in this work, as they will be the problem solvers and changemakers for the coming decades. In this themed issue of SPUR, the authors explore their methods for infusing climate change impacts into classroom and research experiences.

This issue features two practice articles that highlight the importance and impact of integrating climate change work across fields. Sarah Whipple (Colorado State University) and colleagues discuss a US-Australia project integrating sustainable development goals established by the United Nations into courses and project-based learning opportunities. The UN's Sustainable Development Goals-or SDGs-offer a blueprint for a sustainable future. By creating research teams focused on one or more SDGs, the authors bridge the gulf between science and policy as well as study and action. The multidisciplinary, multiinstitutional dimension of the project engenders a broader sense of community among students and faculty, helping students develop the interdisciplinary communication and leadership skills essential for addressing climate change.

Moving to the Arctic region, Arnell Garrett (Delmarva Analysis) and colleagues discuss the Polaris Project, initiated in Siberia and now operated in Alaska. Designed to increase the engagement of historically underrepresented groups in climate science and Arctic research, Garrett and colleagues have developed a robust Research Experiences for Undergraduates (REU) program with the Woodwell
Climate Research Center that introduces students to Arctic science through virtual and in-person meetings, summer fieldwork, and a culminating poster presentation. More than 95 percent of the REU participants come from backgrounds underrepresented in Arctic science. Mentoring by faculty of diverse backgrounds from multiple disciplines is a key part of the program's success. Assessment data document an increase in participant self-efficacy - a trait key to persistence in STEM.

This issue also includes four vignettes that illustrate various undergraduate research applications to climate change. Laura Guertin (Penn State Brandywine) and colleagues discuss the use of a scaffolded audio-narrative project in their introductory earth sciences course that engages students with ongoing projects developing climate solutions and assists in building multiple literacies - informational, digital, and scientific-in the process. To help students understand the complexity of climate change-its roots, impacts, and solutions - Thomas Hickmann (Utrecht University, The Netherlands) has developed a simulation game based on the international climate negotiations he observed at several UN climate change conferences. Hickmann uses this game in courses to help students understand the difficulty in accomplishing collective action on climate change issues and the exacerbating effects of wealth and power disparities and political instability. Robert Nazarian (Fairfield University) discusses his use of data from publicly available computational model intercomparison projects (MIPs) in laboratory work with undergraduates to convey the effects of climate change. These rich data sources allow students to analyze areas of impact to physical, biological, and social systems as well as increase their contextual knowledge, engendering new collaborations with other researchers and nonprofit organizations. Tara Kulkarni (Norwich University) describes the long-standing service-learning component of an introductory environmental engineering course. For almost a decade, students in the course have developed models to demonstrate environmental challenges and solutions, sharing those models with members of the local community such as K-8 students, thus communicating the significance of their research to a much broader audience.

Although the challenges posed by climate change are indeed daunting, these authors provide insight on the effective integration of specific methods and projectslarge and small-into undergraduate research that can help prepare students for the climate change-related challenges they will face in their professional and personal lives. 